\title{
Acute effects of contract-relax proprioceptive neuromuscular facilitation stretching of hip abductors and adductors on dynamic balance
}

\author{
Rafał Szafraniec ${ }^{\text {Corresp., }}{ }^{1}$ ， Krystyna Chromik ${ }^{1}$, Amanda Poborska ${ }^{2}$, Adam Kawczyński ${ }^{1}$ \\ 1 Faculty of Sports Science, University School of Physical Education, Wrocław, Poland \\ 2 Faculty of Natural Sciences and Technology, Karkonosze College, Jelenia Góra, Poland \\ Corresponding Author: Rafał Szafraniec \\ Email address: rafal.szafraniec@awf.wroc.pl
}

Background.Balance control has been shown to play a fundamental role both in everyday life and many athletic activities. An important component of balance control is the somatosensory information gained from muscle spindles and Golgi tendon organs. The changes in the muscle-tendon unit stiffness could alter the ability to detect and respond promptly to changes of an unstable environment. One of the procedures affecting muscle stiffness is stretching, and contract-relax PNF stretching (CRS) is considered as one of the safest and most effective techniques. So far, there are no studies on the impact of CRS of hip adductor and abductor muscles on body balance. These muscle groups are responsible for maintaining mediolateral balance which is of particular interest, since it is more affected by ageing and disease and since its deterioration has been associated with an increased risk of falling. In light of the above, the aim of the present study was to investigate the effects of a single dose of contract-relax proprioceptive neuromuscular facilitation stretching of hip adductors and abductors on mediolateral dynamic balance. Methods.The study involved 45 healthy individuals (age 19-23 years) assigned to the intervention group (IG) or the control group (CG). Balance testing was carried out before (Pre) and immediately after CRS in the intervention group or after 5-minute rest in the control group (Post). There were performed three repetitions of the CRS targeting the adductor and abductor muscles of the hip. Results.Statistically significant differences between Pre and Post condition were observed only in the intervention group. The values of all measured variables defining the body's dynamic balance were significantly lower immediately after the applied CRS, which indicates an improved body balance: Global Index $(p=0.0001)$, total area of sway $(p=0.0001)$, external area of sway $(p=0.00004)$, external time $(p=0.0004)$ and reaction time $(p=0.0003)$. Conclusions. A single dose of contract-relax proprioceptive neuromuscular facilitation stretching of the hip adductor and abductor muscles improved mediolateral dynamic balance. 
1 Acute effects of contract-relax proprioceptive neuromuscular facilitation stretching of hip

2 abductors and adductors on dynamic balance

3

4

5 Rafał Szafraniec ${ }^{1}$, Krystyna Chromik ${ }^{1}$, Amanda Poborska ${ }^{2}$, Adam Kawczyński ${ }^{1}$

6

$7 \quad{ }^{1}$ Faculty of Sports Science, University School of Physical Education, Wrocław, Poland

$8 \quad{ }^{2}$ Faculty of Natural Sciences and Technology, Karkonosze College, Jelenia Góra, Poland

13 Corresponding Author:

14 Rafał Szafraniec ${ }^{1}$

15 al. Ignacego Jana Paderewskiego 35, 51-612 Wrocław, Poland

16 Email address: rafal.szafraniec@awf.wroc.p1 


\section{Abstract}

19 Background. Balance control has been shown to play a fundamental role both in everyday life and many athletic activities. An important component of balance control is the somatosensory information gained from muscle spindles and Golgi tendon organs. The changes in the muscletendon unit stiffness could alter the ability to detect and respond promptly to changes of an unstable environment. One of the procedures affecting muscle stiffness is stretching, and contract-relax PNF stretching (CRS) is considered as one of the safest and most effective techniques. So far, there are no studies on the impact of CRS of hip adductor and abductor muscles on body balance. These muscle groups are responsible for maintaining mediolateral balance which is of particular interest, since it is more affected by ageing and disease and since its deterioration has been associated with an increased risk of falling. In light of the above, the aim of the present study was to investigate the effects of a single dose of contract-relax proprioceptive neuromuscular facilitation stretching of hip adductors and abductors on mediolateral dynamic balance.

32 Methods. The study involved 45 healthy individuals (age 19-23 years) assigned to the intervention group (IG) or the control group (CG). Balance testing was carried out before (Pre) and immediately after CRS in the intervention group or after 5-minute rest in the control group (Post). There were performed three repetitions of the CRS targeting the adductor and abductor muscles of the hip.

37 Results. Statistically significant differences between Pre and Post condition were observed only in the intervention group. The values of all measured variables defining the body's dynamic balance were significantly lower immediately after the applied CRS, which indicates an improved body balance: Global Index $(p=0.0001)$, total area of sway $(p=0.0001)$, external area

41 of sway $(p=0.00004)$, external time $(p=0.0004)$ and reaction time $(p=0.0003)$.

42 Conclusions. A single dose of contract-relax proprioceptive neuromuscular facilitation 43 stretching of the hip adductor and abductor muscles improved mediolateral dynamic balance. 


\section{Introduction}

Balance control has been shown to play a fundamental role both in everyday life and many athletic activities (Grygorowicz et al., 2018; Behm et al., 2015). Postural control is the output of muscle activity that counteracts the destabilizing effects of gravity and inertia in static and dynamic conditions (Duarte \& Sternad, 2008). The ability to maintain balance is based on multiple components that coordinate sensory input and motor output (Li et al., 2018; Dos Santos et al., 2017; Stins et al., 2011; Manor et al., 2010). Due to differences in postural control schemes, the convention has classified balance as either static or dynamic. Static balance is understood as the ability to maintain the body in a fixed upright position with minimal sway over the base of support (Yamagata et al., 2017). In turn, dynamic balance is the ability to maintain postural stability when the body performs a motor activity with a moving base of support (Gürkan et al., 2016). An important component of balance control is the somatosensory information gained from muscle spindles and Golgi tendon organs. Changes in muscle-tendon stiffness could alter the ability to detect and respond promptly to changes of an unstable environment (Herda et al., 2011). On the other hand, flexibility of balance control system is achieved by reweighting sensory inputs based on reliability to balance control in a given situation (Welch \& Ting, 2009).

One of the procedures affecting muscle stiffness is stretching (Taniguchi et al., 2015).

Three common stretching techniques include static stretching, dynamic stretching and proprioceptive neuromuscular facilitation (PNF) (Behm et al., 2015). Two PNF techniques are the most popular: the contract-relax method and the contract-relax-antagonist-contract method (CRAC) (Hindle et al., 2012). Contract-relax stretching (CRS) is considered as one of the safest and most effective stretching techniques. The mechanics of CRS are based on the reflex action of the Golgi tendon organs after isometric tension is applied. Then the motoneuron fields of the activated muscle are attenuated to reduce muscle tension (Hindle et al., 2012). In balance control, changes in a muscle tone may mitigate the effects of a perturbation by changing the mechanical response of the body to perturbation (Welch \& Ting, 2009).

Most studies examined the effects of static stretching on dynamic balance performance but the results are not unequivocal. Some authors reported that static stretching enhances dynamic balance (Nelson et al., 2012; Handrakis et al., 2010; Costa et al., 2009). On the contrary, some studies confirmed the negative effect of static stretching on the body balance 
75 (Chatzopoulos et al., 2014; Han et al., 2014; Lima et al., 2014; Nagano et al., 2006; Behm et al., 76 2004).

Data examining the effects of PNF stretching on balance control are scarce. Ghram et al. 78 (2017) stated that CRS of the quadriceps, hamstrings, anterior tibialis, and calf muscles impaired static balance control in healthy men. In contrast, Ryan et al. (2010) found that CRAC of the hamstrings, plantar and hip flexor muscles improved mediolateral (ML) balance. The authors observed improved stability in ML plane, despite the fact that the flexor muscles of the lower limb were stretched, i.e. the muscles controlling the balance in anteroposterior plane. The ML balance, however, is controlled by hip adductor and abductor muscles (Horak, 2006). We therefore suspect that the stretching of these muscle groups can more significantly improve the ML balance than the stretching of hamstrings or plantar and hip flexors. Ryan et al. suspect that the improvement in ML stability may be because of neurological facilitation from the contractrelax portion, or irradiation overflow from the antagonist-contract phase. To determine which mechanism is more likely, we decided to apply in our research the CRS method, in which there is no the antagonist-contract phase.

Confirmation of the hypothesis that CRS of hip adductors and abductors improves ML balance would have an application value, because efficient body balance is important not only in sports but also in everyday life. Especially mediolateral balance is of particular interest, since it is more affected by ageing and disease and since its deterioration has been associated with an increased risk of falling (Puszczałowska-Lizis et al., 2016; Melzer et al., 2010; Hilliard et al., 95 2008). dose of contract-relax proprioceptive neuromuscular facilitation stretching of hip adductors and abductors on mediolateral dynamic balance.

\section{Material \& Methods}

101 Participants

102 The research protocol was approved by the Senate Commission for Ethics of Scientific Research 103 at the University School of Physical Education in Wrocław. Criteria for exclusion were: impaired 104 vision or hearing, central nervous system disorders, any balance impairment, lower extremity 105 joint or sacroiliac dysfunction, or joint hypermobility syndrome. All included participants $(\mathrm{n}=$ 
10645 , age $19-23$ years) signed informed consent and they were assigned to the intervention group

107 (IG) or the control group (CG). Participant characteristics are presented in Table 1.

108

109 Procedures

110 All subjects visited the laboratory 24 hours before testing session to undergo anthropometric

111 measures and familiarize with the balance test and stretching exercises. They performed the

112 balance test twice and then three repetitions of CRS (only intervention group). Next day, in the

113 testing session, the balance test was carried out before (Pre) and immediately after CRS in the

114 intervention group or after 5-minute rest in sitting position in the control group (Post).

115 Contract-relax proprioceptive neuromuscular facilitation stretching (CRS)

116 Stretching was administered to both limbs targeting the adductor and abductor muscles of the

117 hip, in which the order of treatment was reversed in half of the sample (right and then left limb or

118 vice versa). The CRS involved passive stretch (to the sense of discomfort) of the target muscles,

119 then the 10-second of 50\% maximal voluntary isometric contraction (MVIC) of the same

120 muscles, followed by relaxation $(5 \mathrm{sec})$ and passive movement into further stretch $(5 \mathrm{sec})$. Feland

121 and Marin (2004) found that submaximal contractions (20-60\% MVIC) administered using the

122 CRS method are just as effective as techniques that apply maximal contractions. There were

123 performed three repetitions of the CRS on each muscle group.

124

125 Dynamic balance assessment

126 Mediolateral dynamic balance was measured using the stabilometric platform (Libra, EasyTech,

127 Itlay). The Libra platform is an electronic oscillating balance board that measures mediolateral

128 tilt from $-15^{\circ}$ to $+15^{\circ}$ to an accuracy of $1^{\circ}$. The platform was placed on a level surface using the

129 manufacturer-supplied mat and a computer with dedicated software was connected to the device

130 to register mediolateral tilt. All testing was performed in a quiet, well-lit, temperature-controlled

131 room to minimize external influences. The participant stood barefoot on the balance board and

132 was monitored by personnel for safety precautions in case of loss of balance. Feet were

133 maintained parallel to one another and the upper limbs rested freely along the trunk. In addition,

134 the participants kept their knees extended throughout the test in order to exclude the effects of

135 the knee joint on stability. The task was to maintain balance on the device according to a pre-set

136 level of deviation. During the trial, the participant observed a monitor that showed a sway line 
137 indicative of the amount of deflection from the center line (indicative of zero sway). Two

138 borderlines paralleled the center line and marked the sway threshold (Fig.1). If the participant did

139 not maintain balance on the board, the sway line would deviate from the center line and an audio

140 signal was sounded if one of the borderlines was crossed. The level of difficulty was set to

141 maximum on the device permitting only minimal sway. Dynamic balance was quantified based

142 on the amount of sway registered during the 30-s trial. The angular and temporal values of sway

143 displacement were graphically represented on a stabilogram and used to extract stability

144 outcomes:

145 - Total area of sway (TA) - the summed internal (within the threshold limit) and external

146 (outside the threshold limit) area created by sway line amplitude that deviated from the

$147 \quad$ center line $\left[{ }^{\circ} \mathrm{s}\right]$

148 - External area of sway (EA) - the summed external area created by sway line amplitude

149 outside the threshold limit $\left[{ }^{\circ} \mathrm{s}\right]$

150 - External time (ET) - the summed time when sway line amplitude exceeded the threshold $151 \quad$ limit $[\mathrm{s}]$

- Reaction time (RT) - the longest interval in which sway line amplitude exceeded the threshold limit [s]

- Global index (GI) - a weighted measure of the variables described above calculated on a

\section{Statistical analysis}

158 All data in the text and figures are presented as mean \pm SD. Normal distribution of the data was examined using a Kolmogorov-Smirnov normality test. Initially, mixed-model ANOVA, repeated-measures analysis with one between factor (gender) and one within factor (time) was conducted in the intervention group (IG) to determine if there were differing responses because of gender. There was no significance found between genders, so the male and female scores were combined, and this group's performance was compared with that of the control group (CG) using mixed-model ANOVA, repeated-measures analysis with one between factor (group; IG and CG) and one within factor (time; Pre and Post). Bonferroni post-hoc multiple comparison was performed if a significant main effect was observed. For each ANOVA, partial eta-squared was calculated as measures of effect size. Values of $0.01,0.06$, and above 0.14 were considered as 
168 small, medium, and large, respectively. In the case of non-normality of experimental data (EA

169 and ET), we applied alternative, non-parametric statistical analysis. In that case, a Wilcoxon

170 signed-rank test was utilized to analyze relationships between pairs of related samples (time) and

171 a Mann-Whitney $U$ test was used for analysis of pairs of independent samples (groups).

172 Statistical analyses were performed using Statistica 13.1 software (Dell, USA). The significance

173 level was set at alpha $=0.05$.

174

175 Results

176 Global index

177 Mixed-model ANOVA indicated a significant main effect for groups $(F=5.02, \mathrm{p}=0.03$, partial

178 eta squared $\eta_{\mathrm{p}}{ }^{2}=0.11$, observed power $\left.=0.6\right)$, time $\left(F=6.96, \mathrm{p}=0.01, \eta_{\mathrm{p}}{ }^{2}=0.14\right.$, observed

179 power $=0.7)$ and the group $\mathrm{x}$ time interaction $\left(F=5.74, \mathrm{p}=0.02, \eta_{\mathrm{p}}{ }^{2}=0.12\right.$, observed power $=$

180 0.7). Bonferroni test revealed that GI in the intervention group was significantly lower after CRS

181 (Pre $3.45 \pm 1.34$ vs. Post $2.42 \pm 0.73 ; \mathrm{p}=0.0001$ ). There was no significant difference between

182 Pre and Post in the control group (3.62 \pm 0.98 vs. $3.57 \pm 1.38 ; \mathrm{p}=0.9)$. IG values measured in

183 Pre did not differ $(\mathrm{p}=0.7)$ between IG and CG. IG values in Post condition were lower for IG

184 than $\mathrm{CG}(\mathrm{p}=0.0007)$ (Fig.2).

185 Total area

186 Mixed-model ANOVA showed a significant main effect for groups $\left(F=4.83, \mathrm{p}=0.03, \eta_{\mathrm{p}}{ }^{2}=\right.$

1870.10 , observed power $=0.6)$, time $\left(F=7.23, \mathrm{p}=0.01, \eta_{\mathrm{p}}{ }^{2}=0.14\right.$, observed power $\left.=0.8\right)$ and the 188 group $\mathrm{x}$ time interaction $\left(F=6.72, \mathrm{p}=0.01, \eta_{\mathrm{p}}{ }^{2}=0.14\right.$, observed power $\left.=0.7\right)$. Bonferroni test

189 established a decrease of TA in Post condition in the intervention group (Pre $69.18 \pm 16.45^{\circ} \mathrm{S}$ vs.

190 Post $56.42 \pm 9.69^{\circ} \mathrm{s} ; \mathrm{p}=0.0001$ ) but not in the control group (Pre $71.16 \pm 12.96^{\circ} \mathrm{s}$ vs. Post

$19170.93 \pm 16.20^{\circ} \mathrm{s} ; \mathrm{p}=0.9$ ). There was no significant difference in TA between IG and CG in Pre

$192(\mathrm{p}=0.7)$, however in Post the TA values were lower for IG than CG $(\mathrm{p}=0.0005)$.

193 External area

194 In the intervention group, a Wilcoxon test demonstrated a reduction of EA after the stretching 195 procedure (Pre $4.61 \pm 5.49^{\circ} \mathrm{S}$ vs. Post $\left.1.03 \pm 1.30^{\circ} \mathrm{s} ; \mathrm{p}=0.00004\right)$. In the control group there

196 was no significant difference between Pre and Post condition (Pre 5.86 $\pm 5.94{ }^{\circ}$ s vs. Post $4.11 \pm$

$\left.1975.09^{\circ} \mathrm{s} ; \mathrm{p}=0.7\right)$. Mann-Whitney test revealed that EA was significantly lower in IG as compared 198 to $\mathrm{CG}(\mathrm{p}=0.002)$ only in Post but not in Pre $(\mathrm{p}=0.4)$. 
199

200

201

202

203

204

205

206

207

208

209

210

211

212

213

\section{Discussion}

215

216

217

218

219

220

221

222

223

224

225

226

227

228

229

\section{External time}

\section{Reaction time}

Wilcoxon test showed that ET decreased in the intervention group after stretching (Pre $2.57 \pm$ $1.97 \mathrm{~s}$ vs. Post $1.06 \pm 1.15 \mathrm{~s} ; \mathrm{p}=0.0004)$, however in the control group ET remained almost unchanged (Pre $2.71 \pm 1.64 \mathrm{~s}$ vs. Post $2.71 \pm 2.12 \mathrm{~s} ; \mathrm{p}=0.9$ ). Mann-Whitney test indicated that ET values measured in Pre did not differ $(\mathrm{p}=0.6)$ between IG and CG. ET values in Post condition were lower for IG than CG $(\mathrm{p}=0.003)$.

Mixed-model ANOVA demonstrated a significant main effect for groups $(F=10.29, \mathrm{p}=0.003$, $\eta_{\mathrm{p}}{ }^{2}=0.19$, observed power $\left.=0.9\right)$ and the group $\mathrm{x}$ time interaction $\left(F=5.69, \mathrm{p}=0.02, \eta_{\mathrm{p}}{ }^{2}=\right.$ 0.12 , observed power $=0.7)$. Bonferroni test revealed a decrease of RT in Post condition in the intervention group (Pre $1.14 \pm 0.68 \mathrm{~s}$ vs. Post $0.53 \pm 0.49 \mathrm{~s} ; \mathrm{p}=0.0003$ ) but not in the control group (Pre $1.33 \pm 0.74 \mathrm{~s}$ vs. Post $1.44 \pm 1.09 \mathrm{~s} ; \mathrm{p}=0.7$ ). There was no significant difference in RT between IG and CG in Pre ( $p=0.4)$, however in Post condition the TA was lower for IG than $\mathrm{CG}(\mathrm{p}=0.003)$.

The aim of our study was to investigate the effects of a single dose of CRS of hip adductor and abductor muscles on mediolateral dynamic balance. Analysis of the results showed that single dose of CRS significantly improved mediolateral balance. The improvement of balance was manifested by the magnitude decrease in the postural sway variables, which took place only in the intervention group, but not in the control group. In the intervention group we observed after stretching lower values of balance global index, sway area and reaction time. This proves that after CRS application, the subjects reacted faster to the support surface perturbations, which resulted in a decrease in a sway amplitude. In other words, the dynamic balance of the body was maintained more efficiently.

These results are in line with those reported by Ryan et al. (2010), who investigated the effects of CRAC intervention targeting the hamstrings, quadriceps, iliopsoas and plantar flexor muscles on balance. In their study, each stretch consisted of a passive initial stretch to the point of mild tension or restriction, followed by a 7-second isometric contraction of the target muscle. Then a concentric contraction of the opposite muscle group was performed for 4 seconds. The procedure was conducted 4 times. The authors found that CRAC method improved mediolateral 
230 balance. They indicate the neurological facilitation from the contract-relax stage, or irradiation

231 overflow from the antagonist-contract phase, as potential mechanisms leading to the

232 improvement of body balance. The results of our study, in which the CRS method was used,

233 seem to confirm the first mechanism concerning the neurological facilitation. Young and Elliot

234 argue that a neurological facilitation mechanism may result in a lingering activation of motor

235 units. Furthermore, Ostering et al. state that the CRS technique leads to increased

236 electromyographic (EMG) activity through the isometric contraction, which may help to

237 counterbalance the tendon slack associated with acute static stretching.

238 Ghram et al. (2017) came to completely different conclusions. They stated that CRS of

239 the quadriceps, hamstrings, anterior tibialis, and calf muscles impaired static balance control. In

240 their study, the subjects performed a maximal voluntary isometric contraction (MVIC) of the

241 target muscle during 5-second, followed by 5 -second of relaxation and 5-second of static

242 stretching. We used twice as long (10 seconds) isometric contraction time, but with 50\% MVIC.

243 Both the difference in time of isometric contraction and its strength could influence the

244 achievement of such different results.

245 This study had some limitations. First of all, we did not measure the changes in muscle

246 tone or range of motion. Supplementing the experiment with measurement of physiological

247 properties of the stretched muscles, would allow for a more detailed interpretation of the results.

248 Secondly, we used a healthy and young population, and our results are not generalizable to other

249 populations. Thirdly, we measured only acute effects of CRS so we do not know how long the

250 improvement in balance can be maintained.

251 All the limitations do not negate the fact that our study is of practical importance because

252 it closely simulates the type of interventions used by fitness and rehabilitation professionals. Our

253 results suggest that contract-relax proprioceptive neuromuscular facilitation stretching can be

254 used as one of the means to improve or counteract impairments of body balance.

255 Future research should include the elderly, patients with limited joint range of motion (for

256 whom stretching is particularly indicated), and patients with impaired the visual, vestibular, and

257 somatosensory systems. Futures studies should also include stiffness and electromyographic

258 analyses of the stretched muscles and should evaluate how long the effect is maintained.

\section{Conclusions}


A single dose of contract-relax proprioceptive neuromuscular facilitation stretching of the

hip adductor and abductor muscles improved mediolateral dynamic balance.

\section{References}

Behm DG, Bambury A, Cahill F, Power K. 2004. Effect of acute static stretching on force, balance, reaction time, and movement time. Medicine \& Science in Sports \& Exercise 36:13971402.

Behm DG, Blazevich AJ, Kay AD, McHugh M. 2015. Acute effects of muscle stretching on physical performance, range of motion, and injury incidence in healthy active individuals: a systematic review. Applied Physiology, Nutrition, and Metabolism 41:1-11.

Chatzopoulos D, Galazoulas C, Patikas D, Kotzamanidis C. 2014. Acute effects of static and dynamic stretching on balance, agility, reaction time and movement time. Journal of Sports Science \& Medicine 13:403-409.

Costa PB, Graves BS, Whitehurst M, Jacobs PL. 2009. The acute effects of different durations of static stretching on dynamic balance performance. The Journal of Strength \& Conditioning Research 23:141-147.

Dos Santos DA, Fukuchi CA, Fukuchi RK, Duarte M. 2017. A data set with kinematic and ground reaction forces of human balance. PeerJ 5:e3626.

Duarte M, Sternad D. 2008. Complexity of human postural control in young and older adults during prolonged standing. Experimental Brain Research 191:265-276.

Feland J, Marin H. 2004. Effect of submaximal contraction intensity in contract-relax proprioceptive neuromuscular facilitation stretching. British Journal of Sports Medicine 38:e18-e18.

Ghram A, Damak M, Costa P. 2017. Effect of acute contract-relax proprioceptive neuromuscular facilitation stretching on static balance in healthy men. Science \& Sports 32:e1-e7.

Grygorowicz M, Dzudziński W, Śliwowski R. 2018. Comparison of Static and Dynamic Balance at Different Levels of Sport Competition in Professional and Junior Elite Soccer Players. Journal of Strength \& Conditioning Research doi:10.1519/JSC. 0000000000002476.

Gürkan AC, Demirel H, Demir M, Atmaca EŞ, Bozöyük G, Dane S. 2016. Effects of long-term training program on static and dynamic balance in young subjects. Clinical \& Investigative Medicine 39:31-33.

Han M-J, Yuk G-C, Gak H, Suh S-R, Kim S-G. 2014. Acute effects of 5 min of plantar flexor static stretching on balance and gait in the elderly. Journal of Physical Therapy Science 26:131-133.

Handrakis JP, Southard VN, Abreu JM, Aloisa M, Doyen MR, Echevarria LM, Hwang H, Samuels C, Venegas SA, Douris PC. 2010. Static stretching does not impair performance in active middle-aged adults. The Journal of Strength \& Conditioning Research 24:825-830.

Herda TJ, Costa PB, Walter AA, Ryan ED, Hoge KM, Kerksick CM, Stout JR, Cramer JT. 2011. Effects of two modes of static stretching on muscle strength and stiffness. Medicine and Science in Sports and Exercise 43:1777-1784.

Hilliard MJ, Martinez KM, Janssen I, Edwards B, Mille ML, Zhang Y, Rogers MW. 2008. Lateral balance factors predict future falls in community-living older adults. Archives of Physical Medicine and Rehabilitation 89:1708-1713. 
305

306

307

308

309

310

311

312

313

314

315

316

317

318

319

320

321

322

323

324

325

326

327

328

329

330

331

332

333

334

335

336

337

338

339

340

341

342

343

344

345

346

347

348

349

350

Hindle K, Whitcomb T, Briggs W, Hong J. 2012. Proprioceptive neuromuscular facilitation (PNF): Its mechanisms and effects on range of motion and muscular function. Journal of Human Kinetics 31:105-113.

Horak FB. 2006. Postural orientation and equilibrium: what do we need to know about neural control of balance to prevent falls? Age and Ageing 35:ii7-iil1.

Li Z, Wang X-X, Liang Y-Y, Chen S-Y, Sheng J, Ma S-J. 2018. Effects of the visual-feedbackbased force platform training with functional electric stimulation on the balance and prevention of falls in older adults: a randomized controlled trial. PeerJ 6:e4244.

Lima BN, Lucareli PR, Gomes WA, Silva JJ, Bley AS, Hartigan EH, Marchetti PH. 2014. The acute effects of unilateral ankle plantar flexors static-stretching on postural sway and gastrocnemius muscle activity during single-leg balance tasks. Journal of Sports Science \& Medicine 13:564-570.

Manor B, Costa MD, Hu K, Newton E, Starobinets O, Kang HG, Peng C, Novak V, Lipsitz LA. 2010. Physiological complexity and system adaptability: evidence from postural control dynamics of older adults. Journal of Applied Physiology 109:1786-1791.

Melzer I, Kurz I, Oddsson LI. 2010. A retrospective analysis of balance control parameters in elderly fallers and non-fallers. Clinical Biomechanics 25:984-988.

Nagano A, Yoshioka S, Hay DC, Himeno R, Fukashiro S. 2006. Influence of vision and static stretch of the calf muscles on postural sway during quiet standing. Human Movement Science 25:422-434.

Nelson AG, Kokkonen J, Arnall DA, Li L. 2012. Acute stretching increases postural stability in nonbalance trained individuals. The Journal of Strength \& Conditioning Research 26:3095-3100.

Ostering LR, Robertson RN, Troxel RK, Hansen P. 1990. Differential responses to proprioceptive neuromuscular facilitation (PNF) stretch techniques. Medicine \& Science in Sports \& Exercise 22:106-111.

Puszczałowska-Lizis E, Bujas P, Omorczyk J. 2016. Postural stability in women in the eighth and ninth decades of life. Acta of Bioengineering and Biomechanics 18:115-121.

Ryan EE, Rossi MD, Lopez R. 2010. The effects of the contract-relax-antagonist-contract form of proprioceptive neuromuscular facilitation stretching on postural stability. The Journal of Strength \& Conditioning Research 24:1888-1894.

Stins JF, Roerdink M, Beek PJ. 2011. To freeze or not to freeze? Affective and cognitive perturbations have markedly different effects on postural control. Human Movement Science 30:190-202.

Taniguchi K, Shinohara M, Nozaki S, Katayose M. 2015. Acute decrease in the stiffness of resting muscle belly due to static stretching. Scandinavian Journal of Medicine \& Science in Sports 25:32-40.

Welch TD, Ting LH. 2009. A feedback model explains the differential scaling of human postural responses to perturbation acceleration and velocity. Journal of Neurophysiology 101:32943309.

Yamagata M, Ikezoe T, Kamiya M, Masaki M, Ichihashi N. 2017. Correlation between movement complexity during static standing and balance function in institutionalized older adults. Clinical Interventions in Aging 12:499-503.

Young W, Elliot S. 2001. Acute effects of static stretching, proprioceptive neuromuscular facilitation stretching and maximum voluntary contractions on explosive force production and jumping performance. Research Quarterly for Exercise and Sport 71: 273-279.

Peer] reviewing PDF | (2018:02:25165:3:0:NEW 7 Nov 2018) 
Figure 1

Exemplary stabilogram illustrating sway line amplitude in relation to center and border lines in feedback trial.

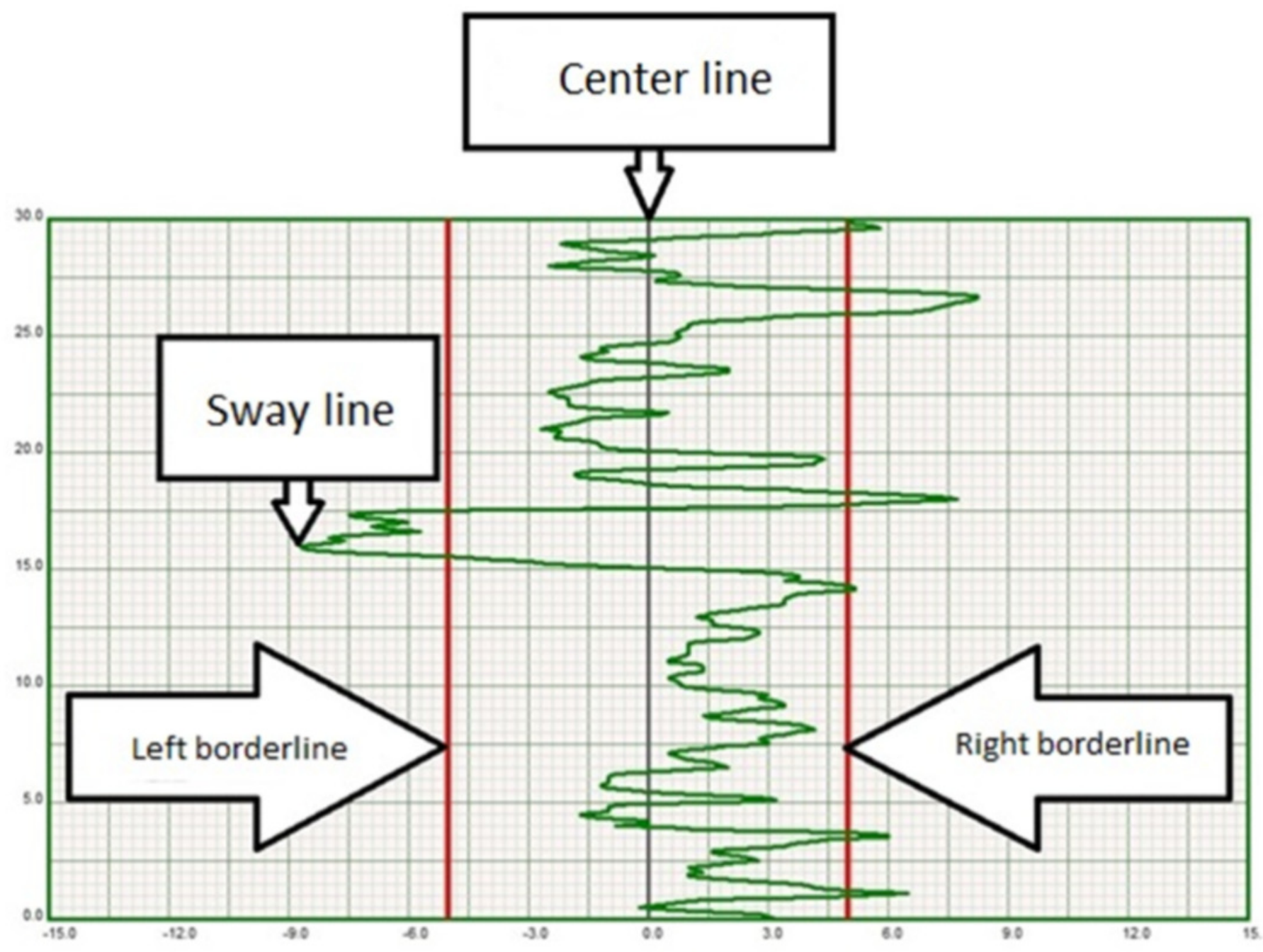


Figure 2 (on next page)

Measurements of body balance before (Pre) and immediately after (Post) CRS in the intervention group (IG) or after 5-minute rest in the control group (CG).

'(A) global index, (B) total area, (C) external area, (D) external time, (E) reaction time. T, time effects; G, group effects; I, interaction effects. $* P<0.001$, significantly different from Pre. \#P $<0.01$, significantly different from CG. 
A Global index (-)

$\mathrm{T}: 0.01(0.14)$

G: $0.03(0.11)$

I: $0.02(0.12)$

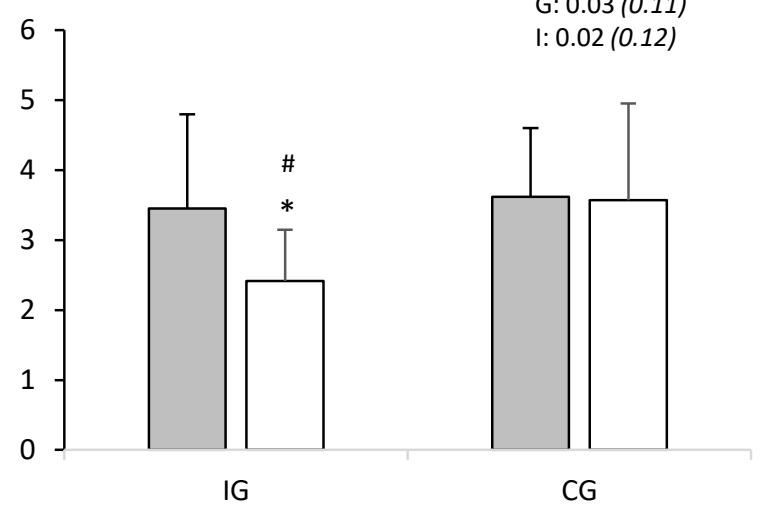

C External area ( $\left.{ }^{\circ} \mathrm{s}\right)$

14
12
10
8
6
4
2
0

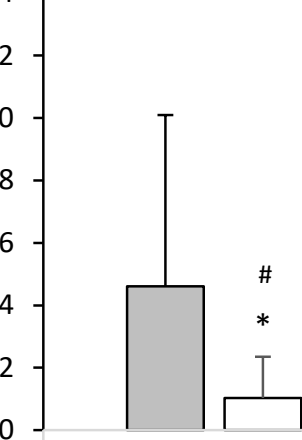

IG

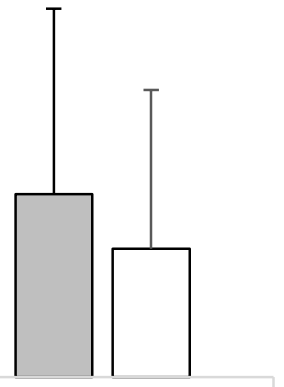

CG
B Total area $\left({ }^{\circ} \mathrm{s}\right)$

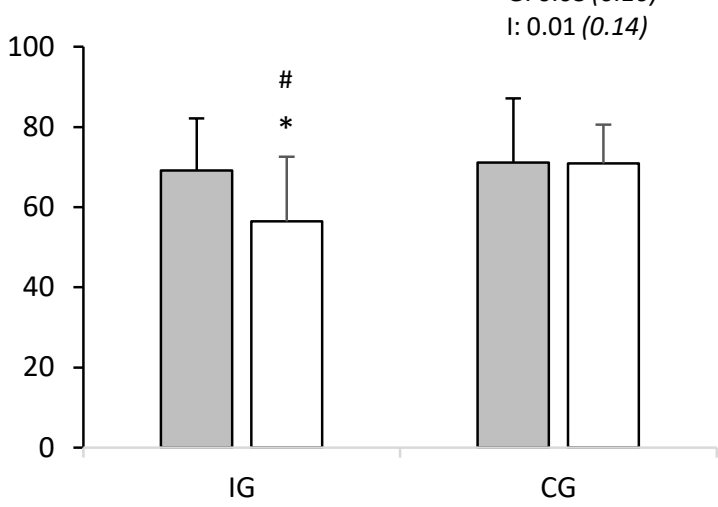

D External time (s)

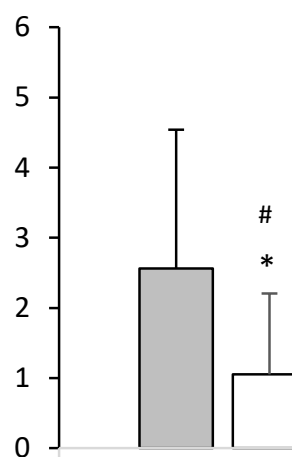

IG
T: $0.01(0.14)$

G: $0.03(0.10)$

I: $0.01(0.14)$

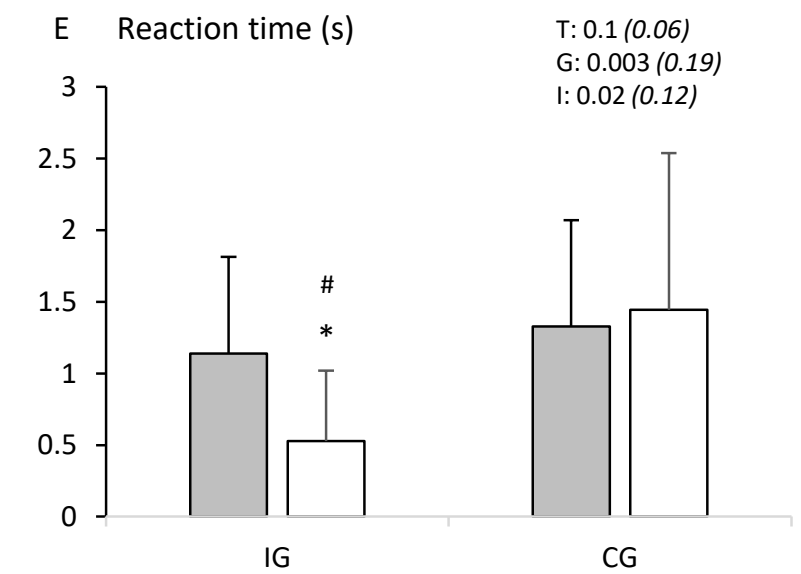




\section{Table 1 (on next page)}

Anthropometric characteristics of the participants (mean $\pm S D$ ). 
1

\begin{tabular}{lllll}
\hline & \multicolumn{2}{c}{ IG } & \multicolumn{2}{c}{ CG } \\
\cline { 2 - 5 } & \multicolumn{1}{c}{ Female $(n=20)$} & Male $(n=11)$ & Female $(n=9)$ & Male $(n=5)$ \\
\hline Age (years) & $20.7 \pm 1.2$ & $21.6 \pm 1.7$ & $20.2 \pm 1.1$ & $21.2 \pm 1.3$ \\
Body height $(\mathrm{cm})$ & $169.4 \pm 6.9$ & $178 \pm 4.9$ & $167.8 \pm 5.7$ & $176.4 \pm 4.1$ \\
Body mass $(\mathrm{kg})$ & $65.8 \pm 9.6$ & $81 \pm 10.8$ & $63.9 \pm 7.8$ & $78.6 \pm 8.9$ \\
BMI $\left(\mathrm{kg} / \mathrm{m}^{2}\right)$ & $23 \pm 3.2$ & $25.5 \pm 2.7$ & $22.9 \pm 2.8$ & $25.3 \pm 2.4$ \\
Fat $(\%)$ & $33.3 \pm 6.0$ & $20.1 \pm 5.1$ & $31.5 \pm 5.1$ & $19.8 \pm 4.8$ \\
\hline
\end{tabular}

2

3 